\title{
MODELO PARA FORMULAR Y EVALUAR PROYECTOS AMBIENTALES EN EMPRESAS LECHERAS
}

\author{
Castignani, M. I. ${ }^{1 ;}$ Rossler, N. ${ }^{1}$; Osan, O. ${ }^{1}$; \\ MAnsilLa, M. E. ${ }^{2}$ \& Rostagno, M. N. ${ }^{3}$
}

\begin{abstract}
RESUMEN
El objeto de este trabajo es presentar una aplicación en Excel, desarrollada por los autores, para evaluar la conveniencia económica y financiera de las inversiones requeridas para implementar un plan de medidas correctivas para el manejo de los efluentes generados en el proceso productivo de empresas lecheras, a fin de mejorar o mitigar los impactos ambientales negativos. El modelo permite determinar los costos asociados a las medidas de mitigación y los ingresos asociados con los daños evitados, incluyéndose ambos en un flujo de cajas del proyecto que se evalúa. Este beneficio se compara con los beneficios obtenidos en la situación vigente (sin proyecto) aplicándose los criterios de evaluación al beneficio incremental: valor actual neto, tasa interna de retorno, periodo de repago. El modelo propuesto funciona como apoyo al proceso de toma de decisiones, generando información sistematizada y de calidad a partir de datos provenientes de empresas reales.
\end{abstract}

Key words: Modelos de simulación, aplicación en excel para el sector agropecuario, Evaluación de proyectos ambientales.

\begin{abstract}
Model to formulate and evaluate environmental projects in dairy farms.

The objective of this paper is to present an application in Excel. It was developed on excel spreadsheets to assess economic and financial convenience of the investments required to implement a corrective action plan for the management of effluents generated in the productive process of dairy farms, in order to improve or mitigate negative environmental impacts. The model allows determining the costs associated with mitigation measures and the incomes associated with the da-
\end{abstract}

1.- Grupo de Economía Agraria Facultad de Ciencias Agrarias (UNL). Kreder 2805. (3080HOF) Esperanza, provincia de Santa Fe. Argentina. Telefax +54 (3496) 426400. Int. 155. Email: mcastign@fca.unl.edu.ar 2.- Alumno de la Maestría en Negocios Agroalimentarios (FCA-FCE UNL), Coordinador del programa Graduado Embajador, UNL. Bv. Pellegrini 2750 - (3000) Santa Fe. Argentina. Tel: +54 (0342) 4571110

3.- Ingeniero Agrónomo. Asesor privado. San Francisco, provincia de Córdoba. Tel: +54 (03564) 15594562. Manuscrito recibido el 23 de octubre de 2019 y aceptado para su publicación el 6 de febrero de 2020.

Castignani, M.I.; Rossler, N.; Osan, O.; Mansilla, M.E. \& Rostagno, M. N. Modelo para formular y evaluar proyectos ambientales en empresas lecheras. FAVE - Ciencias Agrarias 19 (1): 7-16.

CC BY-NC-SA 4.0 (c) (i) (2) 


\section{I. Castignani et al.}

mages avoided. Both are included in a cash flow of the evaluated project. The benefit of the project is compared with the benefits obtained in the current situation of the company without a project applying the evaluation criteria to the incremental benefit: Net present value, internal rate of return and repayment period. The proposed model works to support the decision-making process, generating systematized and quality information based on data from real dairy farms.

Key words: Simulation models, Excel application for the agricultural sector, Ambiental Project evaluation.

\section{INTRODUCCIÓN}

La intensificación de los sistemas lecheros ocurrida desde hace algunos años, obliga a actuar sobre las cuestiones vinculadas con el ambiente, el que se vería perjudicado seriamente de no implementar medidas mitigadoras, remediadoras o compensadoras del impacto negativo generado (1)

Papes y De Prada (2010), mencionan que el problema ambiental causado por los efluentes del tambo puede constituirse en una oportunidad de negocio, considerando el aprovechamiento del mismo, o una amenaza, considerando los efectos ambientales, si no se realiza un tratamiento adecuado del mismo. El manejo apropiado de estos residuos resulta determinante para limitar su efecto negativo sobre el ambiente e indispensable para cumplir con las normativas internacionales que apuntan a producir alimentos con garantías de calidad e inocuidad desde su origen (11). Charlon et al. (2007) muestran que es posible el uso de los residuos del tambo como una fuente de nitrógeno, mejorando la producción y calidad de verdeos, aprovechando un recurso disponible en los tambos. En tanto, Fontanetto et al (2010) concluyen que hay una tendencia a mejorar el contenido de materia orgánica, fósforo y calcio y a mejorar algunas propiedades físicas del suelo mediante el regado con efluentes de tambo.
Costantini y otros (2018) mencionan también el impacto ambiental generado por la emisión de gases de efecto invernadero (GEI) desde el sector agropecuario, como el dióxido de carbono (CO2), el óxido nitroso (N2O) y el metano (CH4), y aclaran que el sector ganadero es un importante emisor de GEI. El Inventario Nacional de GEI muestra que un $49 \%$ de estas emisiones provienen del sector agropecuario, y dentro de este sector el 58\% de los GEI son generados a partir de las distintas fuentes derivadas del ganado bajo pastoreo. No obstante, el Ing. Agr. Ernesto Viglizzo en el panel "Nuevos desafíos" desarrollado en la Jornada de la Mesa de las Carnes realizada en la $131^{\circ}$ Exposición Rural de Palermo, menciona que en la actualidad se miden las emisiones, pero se ignora el secuestro de carbono. Si bien el rumiante emite metano y dióxido de carbono (representan el $65 \%$ de las emisiones ganaderas), hay un secuestro de carbono en las raíces de pastizales, sabanas, praderas y chaparrales que se omiten en las mediciones, desarrollándose solo un inventario y no un balance de carbono (6). En este sentido, según la opinión de expertos, se pueden incorporar algunas prácticas de manejo en el proceso productivo para reducir las emisiones de GEI, como por ejemplo, modificaciones en la dieta de los rumiantes incorporando leguminosas que contribuyen a la degra- 
dabilidad de la fibra, el manejo rotacional de pasturas evitando el sobre pastoreo y trabajando con una adecuada carga animal, el manejo apropiado de los suelos y del estiércol como así también la implementación de sistemas silvopastoriles.

Se recomienda como primer paso en la gestión ambiental, la identificación y evaluación de los impactos sobre el ambiente, positivos o negativos, de las actividades realizadas a fin de definir las medidas correctivas para minimizar o contrarrestar los efectos y desarrollar un plan que contemple inversiones y prácticas que garanticen el cumplimiento de los estándares medioambientales (7).

En este sentido, y aprovechando el avance que se produjo en la comprensión del mundo real con el enfoque sistémico y el modelaje matemático, se ha desarrollado una aplicación en Excel que posibilita la evaluación económica y financiera de las inversiones requeridas para implementar un plan de medidas correctivas para el manejo de efluentes que mejoren, compensen o mitiguen los impactos ambientales negativos causados por el proceso productivo en empresas lecheras, sin importar el tamaño de las mismas.

El objetivo de este trabajo es presentar la aplicación desarrollada.

\section{MATERIALES Y MÉTODOS}

El desarrollo de softwares agropecuarios se encuentra ligado al concepto de modelos matemáticos. Los modelos, son representaciones simplificadas de la realidad; se formulan con un objetivo determinado (5) y permiten analizar una decisión en forma económica apoyando a la toma de deci- siones. Específicamente, un modelo matemático consiste en relaciones matemáticas establecidas en términos de variables, que representan la esencia del problema a resolver (9). En este caso, se formuló para evaluar el cambio de una situación vigente, analizando la variación en la creación de valor futuro que tendría optar por una inversión con relación al valor que se podría esperar si se mantiene la situación actual (9). Es decir, permite determinar, para una empresa en funcionamiento, los costos asociados a las medidas de mitigación y los ingresos asociados con los daños evitados, incluyéndose ambos en un flujo de cajas del proyecto que se evalúa. Este beneficio se compara con los beneficios obtenidos en la situación vigente (sin proyecto) aplicándose los criterios de evaluación al beneficio incremental (10).

Los criterios de evaluación que se utilizan en la aplicación desarrollada son:

* Valor Actual Neto (VAN): mide en términos monetarios los recursos que aporta el proyecto por sobre la rentabilidad exigida en la inversión y después de recuperada toda ella,

* Tasa Interna de Retorno (TIR) que es la tasa de interés en la que el VAN es cero.

La TIR se compara con el costo de oportunidad de la inversión. La distancia entre la TIR hallada y la tasa de descuento utilizada en el cálculo del VAN es una medida de estabilidad de la decisión analizada.

* Periodo de repago: tiempo, en años, que se necesitan para pagar la inversión inicial.

* Máxima inversión que soporta el proyecto evaluado: monto máximo, en términos monetarios, que puede pagarse la inversión sin que el VAN se vuelva negativo.

La aplicación, desarrollada sobre una planilla de Excel permite, además de esti- 
mar la rentabilidad de la inversión (suponiendo que los fondos para llevar adelante la inversión son propios), medir la capacidad del proyecto para enfrentar los compromisos de pago asumidos en un eventual endeudamiento para su realización (10)

Dado que no hay un precio definido en el mercado para la valoración de los efluentes generados en el tambo o los gases contaminantes de efecto invernadero (como el metano), se utilizarán para la valoración de ingresos y/o egresos algunos elementos equivalentes (fertilizantes, equivalentes energéticos, etc.) que si poseen precio de mercado.

\section{RESULTADO Y DISCUSIÓN}

El modelo consta de varias hojas de cálculo, algunas que implican carga de datos (en las celdas de color verdes con letra en color azul) y otras que arrojan los resultados del modelo.

En la imagen 1 se muestra un resumen del modelo.

La arquitectura general y lógica con la que fue desarrollado el modelo es la que se muestra en la imagen 2. Como se observa, el modelo permite evaluar la conveniencia del plan ambiental a través de dos situaciones:
PROYECTO PURO: se evalúan la conveniencia de las inversiones propuestas suponiendo que el $100 \%$ del capital necesario es aportado por la empresa.

PROYECTO CON FINANCIAMIENTO: se evalúan la conveniencia de solicitar un préstamo por una parte o toda la inversión y su efecto sobre la rentabilidad del proyecto.

La conveniencia del PROYECTO PURO surge de comparar los saldos proyectados (ingresos vs egresos) de la situación sin proyecto (que pasaría si no se hacen las inversiones propuestas) con los saldos de la situación con proyecto (esta es la que contempla las inversiones necesarias y los ingresos y egresos derivados de las inversiones).

Respecto al horizonte del proyecto, el modelo permite proyectar a 10 años y hacer todas las inversiones al inicio o en distintos momentos a futuro. El hecho de proponer que el horizonte sea de 10 años es porque, si el proyecto pretende mantenerse en el tiempo, existe una convención que establece utilizar ese periodo de evaluación (10).

A continuación, se presenta cada una de las hojas y los datos necesarios a cargar o los resultados que surgen a partir de la carga de datos.

Imagen 1. Resumen del modelo.

\begin{tabular}{|c|c|c|}
\hline PRESENTACIÓN & HOJAS DE CARGA DE DATOS & HOJAS DE RESULTADOS \\
\hline & Flujo situación sin proyecto & Evaluación proyecto puro \\
\hline & Inversiones & \multirow{2}{*}{$\begin{array}{l}\text { Evaluación proyecto } \\
\text { financiado }\end{array}$} \\
\hline & Flujo situación con proyecto & \\
\hline
\end{tabular}


Hoja $\mathbf{N}^{\circ}$ 1: Flujo Situación sin Proyecto En la imagen 3 se muestra la estimación de efluentes generados en el tambo en la situación sin proyecto.

En la tabla de la imagen 3 se solicita cargar la cantidad de vacas en ordeño para cada uno de los años del horizonte del proyecto. A su vez los litros diarios de efluentes generados por vaca en ordeño y su contenido en Nitrógeno y Fosforo.

Una vez que se completan estos datos, se puede pasar a la siguiente tabla (que también se encuentra en la hoja 1 de la aplica- ción). Aquí se solicita la carga de la cantidad de vacas totales (ordeño + secas), año por año, y la generación anual de metano por vaca. Este dato, generalmente, puede utilizarse en términos medios en cuyo caso se puede encontrar en bibliografía específica o es aportado por organismos de investigación como las Facultades de Ciencias Agrarias o Veterinarias o el INTA. También pueden medirse in situ con herramientas especialmente desarrolladas para esto.

A su vez se deberá completar un precio promedio esperado para el equivalente

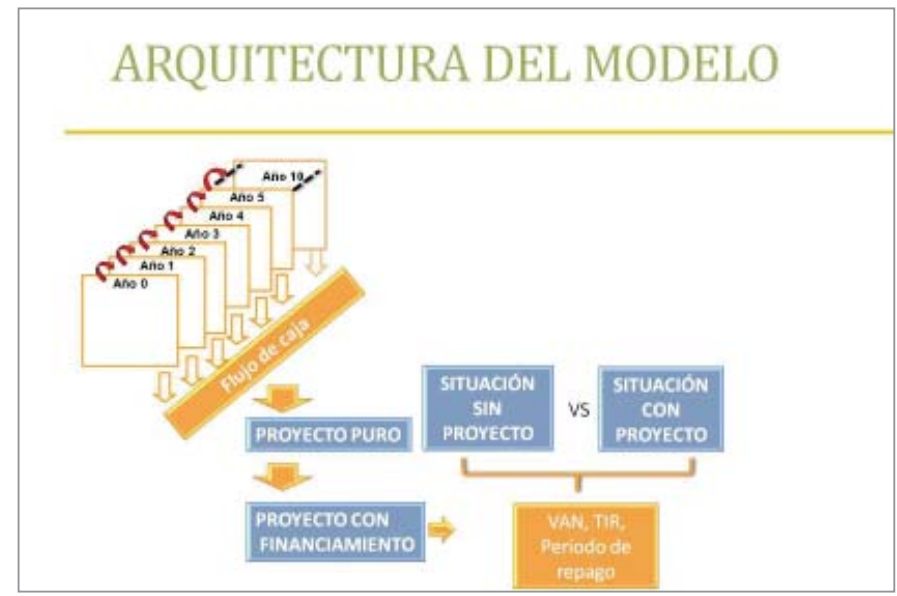

Imagen 2. Arquitectura del modelo

SITUACIÓN SIN PROYECTO: Evaluación de la externalidad negativa en la producción

Estimación de efluentes generados y su composición

- Las celdas de carga de tos tienen fondo verde co

\begin{tabular}{|l|c|c|c|c|c|c|c|c|c|c|}
\cline { 2 - 11 } \multicolumn{1}{c|}{} & año $\mathbf{1}$ & año $\mathbf{2}$ & año $\mathbf{3}$ & año 4 & año $\mathbf{5}$ & año $\mathbf{6}$ & año $\mathbf{7}$ & año 8 & año 9 & año $\mathbf{1 0}$ \\
\hline Vacas en ordeño (cab) & 0 & 0 & 0 & 0 & 0 & 0 & 0 & 0 & 0 & 0 \\
\hline Efluentes generados (I/VO- día) & 0 & 0 & 0 & 0 & 0 & 0 & 0 & 0 & 0 & 0 \\
\hline Efl. Generados (It) & 0 & 0 & 0 & 0 & 0 & 0 & 0 & 0 & 0 & 0 \\
\hline Contenido de Nitrógeno (gr/It efluente) & 0,00 & 0,00 & 0,00 & 0,00 & 0,00 & 0,00 & 0,00 & 0,00 & 0,00 & 0,00 \\
\hline Contenido de Fósforo (gr/It efluente) & 0,00 & 0,00 & 0,00 & 0,00 & 0,00 & 0,00 & 0,00 & 0,00 & 0,00 & 0,00 \\
\hline Nitrógeno total generado (Kg) & 0 & 0 & 0 & 0 & 0 & 0 & 0 & 0 & 0 & 0 \\
\hline Fósforo total generado (Kg) & 0 & 0 & 0 & 0 & 0 & 0 & 0 & 0 & 0 & 0 \\
\hline Equivalente Urea (Kg) & 0 & 0 & 0 & 0 & 0 & 0 & 0 & 0 & 0 & 0 \\
\hline Equivalente Superfosfato Triple SPT (Kg) & 0 & 0 & 0 & 0 & 0 & 0 & 0 & 0 & 0 & 0 \\
\hline
\end{tabular}

Imagen 3. Hoja $N^{\circ}$ 1: flujo situación sin proyecto.

Revista FAVE - Ciencias Agrarias 19 (1) 2020 | 
energético del gas metano (gasoil o gasolina, propano, etc.), de la urea y del superfosfato triple (SPT) (Imagen 4)

Se utilizan los equivalentes urea (para valorar el nitrógeno) y superfosfato triple (para valorar el fósforo) porque son fertilizantes muy utilizados en los sistemas lecheros del centro de la provincia de Santa Fe.

Los precios solicitados permitirán valorar la contaminación por generación de metano (CH4), en el caso del equivalente energético del metano (como gasoil o gasolina, gas propano, energía eléctrica, etc.), o los ahorros estimados en equivalentes de fertilizantes por la concentración de nitrógeno y fósforo existente en los efluentes que pueden ser utilizados en lotes de la explotación o vendidos a un tercero generándose así un ingreso económico.

En la imagen 5, además de la valorización de la contaminación por liberación de metano al ambiente, el usuario del modelo podrá cargar otras situaciones valoradas de contaminación no previstas.

De esta manera finaliza la carga de la hoja 1 del modelo.

Hoja $\mathbf{N}^{\circ}$ 2: Inversiones

En la hoja 2, el usuario debe cargar todas las inversiones necesarias para el plan de medidas correctivas (Imagen 6)

Para completar esta hoja, es importante definir previamente el momento en el que se realizará cada una de las inversiones. $\mathrm{Al}$ respecto, el momento 0 es el momento previo al inicio del proyecto. Las inversiones pueden ser realizadas en cualquier momento a lo largo de la vida del proyecto, en función de lo programado.
Las inversiones pueden ser tanto de activos tangibles (construcciones, adquisición de equipamiento, vehículos, etc.) o intangibles (adquisición de licencias, capacitación del personal, etc.) (4) A modo de resumen, al final de cada columna de la tabla de la imagen 6 , el modelo calcula el valor total de las inversiones correspondientes al momento 0 y a cada uno de los años del proyecto.

Finalizada la carga de las inversiones, se procederá a completar la hoja de cálculo $\mathrm{N}^{\circ}$ 3: Flujo de situación con proyecto.

Hoja No 3: Flujo Situación con Proyecto El flujo de la situación con proyecto está compuesto por la proyección de ingresos, inversiones totales y egresos. Como resultado se obtiene el saldo anual proveniente de la diferencia entre ingresos y egresos e inversiones (Imagen 7)

Los ingresos derivados del plan de medidas correctivas están compuestos por el ahorro estimado en fertilizantes a partir de la inversión en un sistema de tratamiento de efluentes, los ingresos adicionales que puedan lograrse por evitar la emisión parcial de metano y, además, brinda la posibilidad de cargar manualmente otros ingresos que uno considere pertinentes al proyecto. Además, deberá cargar anualmente los gastos de mantenimiento y reparaciones asociados al plan y a las inversiones y tiene la posibilidad de cargar otros ingresos y egresos generados por la implementación del plan. Finalmente se debe cargar el porcentaje de valor residual de las inversiones o valor de desecho de los activos remanentes al final del periodo de evaluación (10). 
Modelo para formular y evaluar proyectos ambientales

Estimación de gases de efecto invernadero generados

\begin{tabular}{|l|c|c|c|c|c|c|c|c|c|c|}
\cline { 2 - 11 } \multicolumn{1}{c|}{} & año $\mathbf{1}$ & año 2 & año 3 & año $\mathbf{4}$ & año $\mathbf{5}$ & año $\mathbf{6}$ & año $\mathbf{7}$ & año 8 & año 9 & año $\mathbf{1 0}$ \\
\hline Vacas en ordeño + vacas secas (cab) & 0 & 0 & 0 & 0 & 0 & 0 & 0 & 0 & 0 & 0 \\
\hline Generación de Metano (CH4) (kg/vaca año) & 0 & 0 & 0 & 0 & 0 & 0 & 0 & 0 & 0 & 0 \\
\hline Generación de Metano (CH4) (kg/año) & 0 & 0 & 0 & 0 & 0 & 0 & 0 & 0 & 0 & 0 \\
\hline Equivalente energético del gas metano (//año de gasoil) & 0 & 0 & 0 & 0 & 0 & 0 & 0 & 0 & 0 & 0 \\
\hline
\end{tabular}

Precios

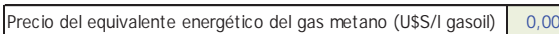

\begin{tabular}{|l|l|}
\hline Precio Urea $(\mathrm{U} \$ \mathrm{~S} / \mathrm{kg})$ & 0,00 \\
\hline Precio Supets & 0,00 \\
\hline
\end{tabular}

\begin{tabular}{|l|l|}
\hline Precio Superfosfato Triple $(\mathrm{U} \$ \mathrm{~S} / \mathrm{kg})$ & 0,00 \\
\hline
\end{tabular}

Imagen 4. Hoja $N^{\circ}$ 1: Estimación de emisión de metano y carga de precios para valoración de ingresos y egresos.

FLUJO DE LA SITUACIÓN SIN PROYECTO

\begin{tabular}{|c|c|c|c|c|c|c|c|c|c|c|}
\hline & \multirow{2}{*}{\multicolumn{10}{|c|}{ U\$S }} \\
\hline \multirow{2}{*}{ Valor de la contaminación por generación de Metano $(\mathrm{CH} 4)$} & & & & & & & & & & \\
\hline & 0 & 0 & 0 & 0 & 0 & 0 & 0 & 0 & 0 & 0 \\
\hline 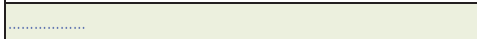 & 0 & 0 & 0 & 0 & 0 & 0 & 0 & 0 & 0 & 0 \\
\hline ……....... & 0 & 0 & 0 & 0 & 0 & 0 & 0 & 0 & 0 & 0 \\
\hline \begin{tabular}{|c|}
$\ldots \ldots \ldots \ldots \ldots \ldots \ldots$ \\
\end{tabular} & 0 & 0 & 0 & 0 & 0 & 0 & 0 & 0 & 0 & 0 \\
\hline Total costo de las externalidades & 0 & 0 & 0 & 0 & 0 & 0 & 0 & 0 & 0 & 0 \\
\hline
\end{tabular}

Imagen 5. Hoja $N^{\circ}$ 1: flujo situación sin proyecto.

\section{SITUACIÓN CON PROYECTO}

FLUJO DE CAJA DEL PROYECTO PURO

I tan celian de cares de tententevertordo

\begin{tabular}{|c|c|c|c|c|c|c|c|c|c|c|c|c|}
\hline & \multicolumn{11}{|c|}{ U\$S } \\
\hline & & momento 0 & año 1 & año 2 & año 3 & año 4 & año 5 & año 6 & año 7 & año 8 & año 9 & año 10 \\
\hline \multicolumn{13}{|l|}{ INGRESOS* } \\
\hline Por ahorro estimado en equivalente UREA & & & 0 & 0 & 0 & 0 & 0 & 0 & 0 & 0 & 0 & \\
\hline Por ahorro estimado en equivalente SPT & & & 0 & 0 & 0 & 0 & 0 & 0 & 0 & 0 & 0 & 0 \\
\hline Por disminución de emisión de metano & $0 \%$ & & 0 & 0 & 0 & 0 & 0 & 0 & 0 & 0 & 0 & 0 \\
\hline Valoración carbono orgánico & & & 0 & 0 & 0 & 0 & 0 & 0 & 0 & 0 & 0 & 0 \\
\hline Ahorro de agua & & & 0 & 0 & 0 & 0 & 0 & 0 & 0 & 0 & 0 & 0 \\
\hline INVERSIONES TOTALES & & 0 & 0 & 0 & 0 & 0 & 0 & 0 & 0 & 0 & 0 & 0 \\
\hline \multicolumn{13}{|l|}{ EGRESOS } \\
\hline Gastos de mantenimiento y reparaciones & & & 0 & 0 & 0 & 0 & 0 & 0 & 0 & 0 & 0 & 0 \\
\hline Egresos por liberación no controlada de metano & $100 \%$ & & 0 & 0 & 0 & 0 & 0 & 0 & 0 & 0 & 0 & 0 \\
\hline Monitoreo y otros egresos & & & 0 & 0 & 0 & 0 & 0 & 0 & 0 & 0 & 0 & 0 \\
\hline Otros egresos & & & 0 & 0 & 0 & 0 & 0 & 0 & 0 & 0 & 0 & 0 \\
\hline VALOR RESIDUAL & $0 \%$ & & & & & & & & & & & 0 \\
\hline SALDO & & 0 & 0 & 0 & 0 & 0 & 0 & 0 & 0 & 0 & 0 & 0 \\
\hline
\end{tabular}

*Ahorros logrados por implementación del proyecto

Imagen 6. Hoja $N^{\circ}$ 3: Flujo de caja del proyecto puro.

Revista FAVE - Ciencias Agrarias 19 (1) 2020 | 
Hoja No 4: Evaluación Proyecto Puro

En esta hoja el único dato por cargar es la tasa de descuento, esto es, la tasa de interés que se utilizará para descontar los flujos generados (Imagen 7).

El modelo brinda como resultado el VAN, la TIR y el periodo de repago. Además, se puede estimar la inversión máxima soportada por el proyecto, sensibilizando el valor de las inversiones en la Hoja 2, y cargarlo manualmente en la celda B15 de la hoja 4 para que queden los criterios completos. A partir de ellos es que se realizan las interpretaciones y se concluye acerca de la factibilidad técnica y viabilidad económica del proyecto.

Hoja No 5: Evaluación Proyecto Financiado

En esta hoja (Imagen 8) se hace una evaluación del proyecto considerando aportes de dinero no provenientes del empresario. Deberán cargarse el monto recibido por el préstamo y el momento y, a su vez, la devolución del mismo, esto es valor anual de la devolución considerando la cuota o las cuotas totales a pagar por año. Es necesario recordar que, las cuotas, incluyen la devolución del capital prestado, los intereses y todos los gastos asociados. Deberá cargar nuevamente la tasa de descuento que se aplicará sobre los saldos anuales.

Como resultados el modelo calcula VAN, TIR y el período de repago, a partir de los flujos. Además, muestra el efecto de la financiación sobre la rentabilidad del proyecto.

A partir del uso del modelo presentado, se pueden emular varias alternativas estratégicas de inversión, verificando su factibilidad técnica, económica y financiera. A través de la variación de los valores de algunas de las variables utilizadas para la evaluación (precios, cantidad de animales, cantidad y calidad de efluentes, tasa de descuento, etc.) se analiza la sensibilidad del proyecto, a partir de la cuantificación del impacto sobre los resultados económicos y financieros del proyecto.

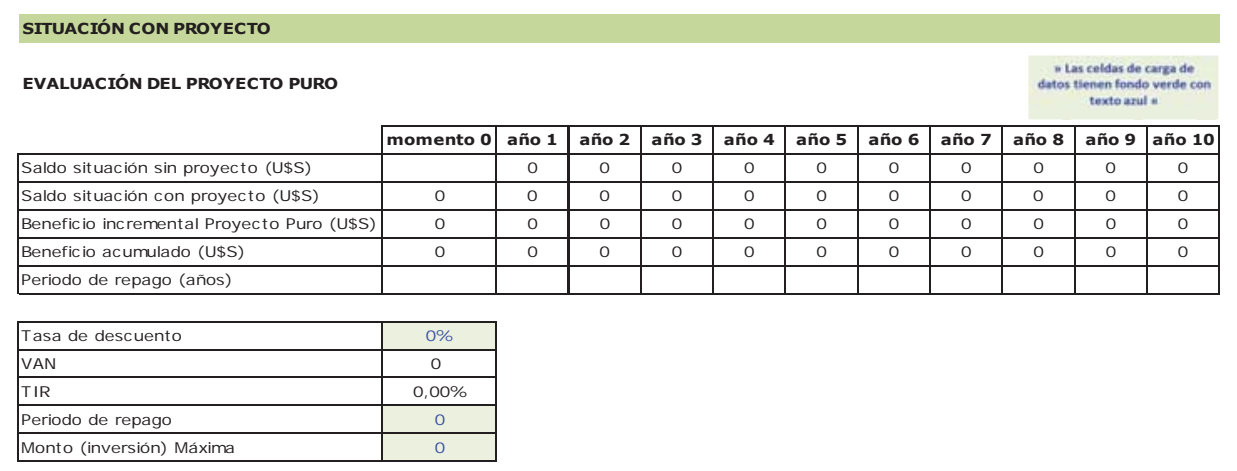

Imagen 7. Hoja $N^{\circ}$ 4: Evaluación del proyecto puro. 
SITUACIÓN CON PROYECTO

EVALUACIÓN DEL PROYECTO FINANCIADO

- Las certibu de carja de dotos tienen fondo verde
terto asul a

\begin{tabular}{|c|c|c|c|c|c|c|c|c|c|c|c|}
\hline & \begin{tabular}{|l|} 
momento 0 \\
\end{tabular} & año 1 & año 2 & aก̃o 3 & año 4 & año 5 & año 6 & aก̃o 7 & año 8 & aก̃o 9 & año 10 \\
\hline Beneficio incremental Proyecto Puro $(\mathrm{U} \$ \mathrm{~S})$ & 0 & 0 & 0 & 0 & 0 & 0 & 0 & 0 & 0 & 0 & 0 \\
\hline Préstamos recibidos (U\$S) & 0 & 0 & 0 & 0 & 0 & 0 & 0 & 0 & 0 & 0 & 0 \\
\hline Devolución del préstamo (amortización + intereses) (U\$S) & 0 & 0 & 0 & 0 & 0 & 0 & 0 & 0 & 0 & 0 & 0 \\
\hline Saldo (U\$S) & 0 & 0 & 0 & 0 & 0 & 0 & 0 & 0 & 0 & 0 & 0 \\
\hline Saldo acumulado (U\$S) & 0 & 0 & 0 & 0 & 0 & 0 & 0 & 0 & 0 & 0 & \\
\hline Periodo de repago (años) & & & & & & & & & & & \\
\hline
\end{tabular}

\begin{tabular}{|l|c|}
\hline Tasa de descuento & $0 \%$ \\
\hline VAN (U\$S) & 0 \\
\hline TIR & $0,00 \%$ \\
\hline Periodo de repago (años) & 0 \\
\hline
\end{tabular}

Apalancamiento

Cambio en la rentabilidad

\begin{tabular}{|l|l|}
\hline Rentabilidad adicional & $0,00 \%$ \\
\hline
\end{tabular}

Imagen 8. Hoja $N^{\circ}$ 5: Evaluación del proyecto financiado

\section{CONCLUSIONES}

El proceso de toma de decisiones se ve favorecido cuando existe información de calidad disponible. La modelización es una herramienta útil para apoyar este proceso, porque permite comprender el funcionamiento de sistemas reales y obtener información sobre bases racionales.

El modelo desarrollado es una herramienta útil para apoyar a profesionales de las ciencias agropecuarias que asesoran a empresarios del sector y evalúan alternativas para mitigar el efecto de las externalidades generadas durante el ciclo productivo y que, finalmente, serán consideradas en el proceso de toma de decisiones que se realiza en las empresas lecheras. Además, este modelo podría ser utilizado como una herramienta adicional en el proceso de enseñanza - aprendizaje de alumnos de carreras afines a las ciencias agropecuarias o a la administración rural, permitiendo enfocarse en la interpretación de resultados y el impacto de la modificación de determinadas variables en este tipo de sistemas.

\section{BIBLIOGRAFÍA}

1.- CASTIGNANI, M. I.; ROSSLER, N., OSAN, O.; SUERO, M.; TRAVADELO, M.; MAINA, M. Y BRIZI, M.C. 2016. Evaluación del impacto de la tecnología y el cuidado del ambiente sobre los resultados físicos y económicos en sistemas lecheros, a través de un modelo de mediano plazo. (pp. 305-326). En: Vicien, C.; S. Pena De Ladaga; G. Petri (Compiladores). Modelización económica en el sector agropecuario VI. Ed. Orientación Grafica. Buenos Aires, Argentina.

2.- COSTANTINI, A.; PEREZ, M.G. BUSTO; M. GONZÁLEZ; F. COSENTINO, V.; ROMANIUK, R. Y TABOADA, M.A. 2018. Emisiones de gases de efecto invernadero en la producción ganadera. Ciencia e investigación 68:5.

3.- CHARLON, V.; ROMERO, L.; CUATRÍN, A. Y TAVERNA, M. 2007. Utilización de residuos orgánicos en el rendimiento y la calidad de un cultivo de avena. Revista Argentina de Producción Animal 27:1. 
4.- FONTANETTO, H.; GAMBAUdO, S.; 8.- PAPES, H.G. Y DE PRADA, J.D. 2010. CHARLÓN, V.; TAVERNA, M.; IMHO-

FF, S. Y ZEN, O. 2010. Manejo y utilización de los efluentes de sistemas ganaderos. Dirección: http://www.engormix.com/MA-ganaderia-leche/manejo/articulos/manejo-utilizacion-efluentes-sistemas-t2817/124-p0. htm. Fecha de consulta: 20/01/2020.

5.- FRANK, R.G. 2010. La optimización en la empresa agraria con programación lineal. Universidad de Buenos Aires. Buenos Aires. Argentina. $464 \mathrm{p}$.

6.- INFOCAMPO. Noticias de campo. Aseguran que Argentina tiene "crédito de carbono” en la producción ganadera. Dirección: https://www.infocampo.com.ar/ aseguran-que-argentina-tiene-credito-de-carbono-en-la-produccion-ganadera/ Fecha de consulta: 20/01/2020.

7.- KVOLEK, C. 2018. Gestión ambiental pecuaria. Orientación Gráfica Editora. Buenos Aires. Argentina. 152p.

Impacto económico de alternativas de manejo de efluentes de bovino de leche, Córdoba, Argentina. XLI Reunión Anual de la Asociación Argentina de Economía Agraria. Potrero Funes. San Luis. Argentina. 19 pp.

9.- PENA, S. 2006. Toma de decisiones en el sector agropecuario: herramientas de investigación operativa aplicadas al agro. FAUBA. Buenos Aires. Argentina. 320 p.

10.- SAPAG CHAIN, N. 2001. Evaluación de proyectos de inversión en la empresa. Prentice Hall. Buenos Aires. Argentina. 416 pp.

11.- TAVERNA, M. 2006. El manejo de efluentes. E.E.A INTA Rafaela. Dirección: http:// www.produccion-animal.com.ar/ Fecha de consulta: 25/01/2020. 